BACTERIAL PHYSIOLOGY

\title{
Setting the speed of translation
}

elongating
ribosomes
pause at
internal
SD-like
sequences
during
translation

The rate of translation in bacteria varies according to the sequence of the mRNA template. One widely held hypothesis predicts that synonymously coded mRNAs are translated at different rates because transient ribosome stalling occurs at codons that are decoded by rare tRNAs. However, there have been suggestions that tRNA abundance is not the sole factor limiting translation rate in vivo. Weissman and colleagues now reveal that sequences within the mRNA coding region that resemble the Shine-Dalgarno (SD) sequence are the predominant cause of translational pausing in bacteria.

The authors used ribosome profiling, a recently developed technique based on deep sequencing of ribosome-protected mRNA fragments, to map the genome-wide distribution of ribosomes along the mRNAs of Escherichia coli and Bacillus subtilis. This approach allowed them to infer the transit time of ribosomes on different

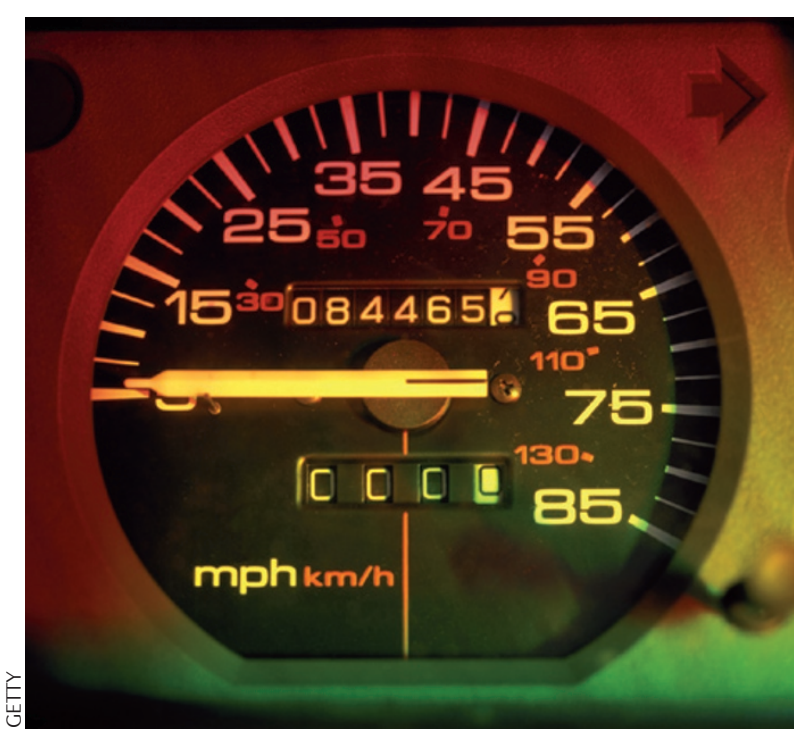

templates. They observed that ribosome density varied more than tenfold across protein coding regions and, unexpectedly, the identity of codons in the ribosomal A-site (where decoding by tRNAs occurs) had only a minor effect on average ribosome occupancy when the cells were cultivated in nutrient-rich conditions.

To better understand the mRNA sequence features that contribute to slow translation, the authors examined the correlation between specific trinucleotide sequences in the coding region and ribosome occupancy. The canonical SD sequence is a purine-rich motif that hybridizes to the complementary anti-SD sequence of the 16S rRNA during translation initiation. In both E. coli and B. subtilis, a strong correlation was observed for coding sequences that resembled the SD motif (SD-like sequences), and this was enhanced when such sequences were located upstream of the ribosomal A-site, at positions corresponding to the optimal distance of a canonical SD sequence from the start codon.

Excess ribosome occupancy may be due to either authentic pausing of elongating ribosomes or attempted internal initiation by newly formed ribosomes. To distinguish between these two possibilities, an orthogonal ribosome (O-ribosome) system was used in which wild-type ribosomes and $\mathrm{O}$-ribosomes are co-expressed. O-ribosomes are engineered with an altered anti-SD and exclusively translate mRNAs that contain a corresponding orthogonal SD (O-SD) sequence. Because wildtype ribosomes do not hybridize to the O-SD sequence, the authors were able to specifically identify pausing of O-ribosomes at internal sequences resembling the $\mathrm{O}-\mathrm{SD}$ motif in the lacZ mRNA. Unlike with wild-type ribosomes, density peaks were detected at O-SD motifs when O-ribosomes were used, providing evidence that elongating ribosomes pause at internal SD-like sequences during translation.

The speed of translation is a major factor controlling bacterial growth rate. Thus, the authors analysed the global distribution of internal SD-like sequences in E. coli and found that they were infrequent in protein-coding genes, leading to the suggestion that selection against SD-like sequences drives codon choice in bacteria. As predicted, purine-rich codons are generally under-represented, and codon pairs resembling SD sequences are rare in the genomes of both E. coli and B. subtilis. Although these sequences appear to be counter-selected, codon choice is restrained by the particular amino acid requirements of the encoded proteins, and SD-like sequences were estimated to be responsible for approximately $70 \%$ of strong ribosomal pauses.

These findings necessitate a re-evaluation of our understanding of translation rate control. Moreover, the observed biased selection against these sequences in protein encoding genes, in two divergent species, suggests that avoidance of anti-SDmediated stalling may be more important than tRNA abundance in driving codon choice in bacteria. These findings lay the foundation for exploring how other cellular processes, such as transcription and co-translational protein folding, are influenced by the speed of translation. Christina Tobin Kåhrström

ORIGINAL RESEARCH PAPER Li, G.-W., Oh, E. \& Weissman, J. S. The anti-Shine-Dalgarno sequence drives translational pausing and codon choice in bacteria. Nature 28 Mar 2012 (doi:10.1038/ nature10965) 